\section{ARE COMPLICATIONS DURING PREGNANCY IN WOMEN WITH MECHANICAL HEART VALVES INFLUENCED BY THEIR CHOICE OF ANTICOAGULATION?}

${ }^{1}$ Helen Parry*, ${ }^{2}$ Kate English, ${ }^{3}$ James Oliver, ${ }^{2}$ Damien Cullington, ${ }^{2}$ Etienne Ciantar, ${ }^{4}$ Nigel Simpson, ${ }^{5}$ Sarah Winfield, ${ }^{2}$ Derek Norfolk. 'Leeds Teaching Hospital; ${ }^{2}$ Leeds Teaching Hospitals; ${ }^{3}$ Leeds Teaching hospitals; ${ }^{4}$ Leds Teaching hospitals; ${ }^{5}$ Leeds teaching Hospitals

\subsection{6/heartjnl-2017-311726.75}

Introduction Anticoagulation during pregnancy in women with mechanical valves is complex. Strategies include warfarin or low-molecular weight heparin (LMWH) throughout or LWMH during first trimester then warfarin until delivery. Warfarin poses risks of teratogenicity and fetal haemorrhage. However, LMWH poses increased thrombotic risk and frequent difficulties maintaining therapeutic peak and trough anti-Xa levels. We aimed to assess the complication rate in pregnant women with mechanical valves and whether anti-Xa levels were maintained within the recommended range with LMWH

Methods All pregnant women with mechanical valves under the ACHD team at Leeds General Infirmary were identified 2001- 2016. Medical records and blood results were analysed, identifying thrombotic and haemorrhagic complications, pregnancies leading to delivery of a healthy child and efficacy of anti-factor Xa monitoring.

Results The 12 patients identified had 25 pregnancies; 12 ultimately delivered a healthy child, 2 of which had no complications. Thrombotic events complicated 6 pregnancies, haemorrhage complicated 5 pregnancies and 1 pregnancy was complicated by both (including all pregnancies/miscarriages). Three pregnancies underwent planned termination, 7 miscarried pre-8 weeks gestation with 3 intra-uterine deaths. Ten patients used LMWH in the first trimester only and 5 used LMWH throughout pregnancy. The graph shows peak anti-factor $\mathrm{Xa}$ levels measured closest to complication development or average peak level if there were no complications (where measurements were available). The peak target was 1.0-1.2.

Conclusions Pregnancy in women with ACHD and mechanical valves carried significant risk of haemorrhage and thrombosis, regardless of anti-coagulation strategy. Anti-Xa levels were generally not maintained within target range.

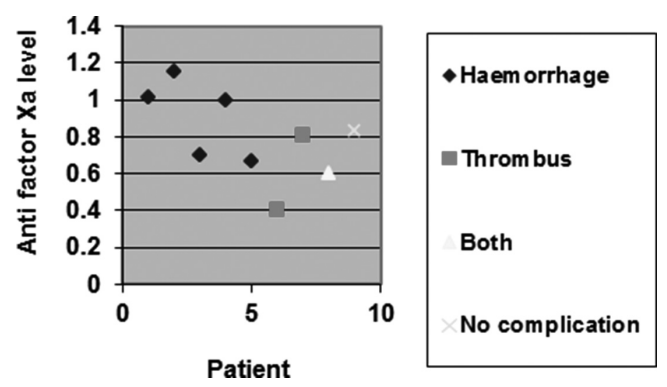

Abstract 76 Figure 1 Graph shows patients rarely have anti factor xa levels in range.

\section{IMPLANTABLE CARDIOVERTER-DEFIBRILLATOR IN PREGNANCY- DO WE KNOW WHAT TO DO?}

${ }^{1}$ Polyvios Demetriades*, ${ }^{2}$ Hsu Chong, ${ }^{3}$ Sara Thorne, ${ }^{1}$ Howard Marshall, ${ }^{1} J o s e p h$ DeBono, ${ }^{2}$ Katie Morris, ${ }^{3}$ Hudsmith Lucy. ${ }^{1}$ Cardiology Department, Queen Elizabeth Hospital; ${ }^{2}$ Birmingham Women's Hospital; ${ }^{3}$ Department of Adult Congenital Heart Disease, Queen Elizabeth Hospital

\subsection{6/heartjnl-2017-311726.76}

Introduction The number of women of reproductive age with an implantable cardioverter-defibrillator (ICD) is increasing, many with Adult Congenital Heart Disease or inherited cardiomyopathy. Safe management of these patients during pregnancy, labour and delivery is paramount. Guidelines exist for ICD management in non-obstetric surgical procedures but there is little information for outcomes in pregnancy or management of ICDs in elective and emergency obstetric surgery. AIMS To investigate knowledge of health care workers about the management of ICDs in pregnancy, prior to drafting new guidelines.

Methods Paper and electronic copies of the focused questionnaire were distributed to healthcare workers within the departments of cardiology, anaesthetics and obstetrics at a large quaternary cardiothoracic centre and separate but co-located tertiary obstetric unit

Results 87 responses were returned (58\% Obstetrics, 33\% Cardiology, 9\% Anaesthetics). Most respondents were trainees (37\%) followed by consultants (28\%), midwives (20\%), physiologists (11\%) and others (4\%). Most (59\%) of the cardiology team had treated a pregnant woman with an ICD. Overall, only $30 \%$ of respondents were confident about inactivating an ICD in an emergency using a magnet (4\% obstetrics, 79\% cardiology, $12 \%$ anaesthetists) and only $20 \%$ were aware of the magnet's location in delivery suite. Most respondents (53\%) were unsure about when to inactivate an ICD (70\% of obstetric team, 32\% cardiologists, 50\% cardiac physiologists) and whether to inactivate in an emergency Caesarian section. When seeking guidance, most respondents (64\%) would contact the cardiology team, 46\% the cardiac physiologists and $13 \%$ the anaesthetist. However, $88 \%$ of cardiologists said they would contact the physiologist whereas most (83\%) physiologists would contact the cardiologist.

Conclusions Knowledge of ICD use in pregnancy was poor amongst those surveyed. It is of concern that most obstetricians would call the cardiology $\mathrm{SpR}$ or physiologists, many of whom are not confident in the emergency management of ICDs in pregnancy. We plan to introduce guidelines, signposting stickers on obstetric notes and training involving the cardiology, obstetrics and anaesthetic departments regarding its use and then re-assess knowledge to optimise patient safety. There is an urgent need for coordinated national guidelines and registries on the management of implantable cardiac devices in pregnancy. 